\title{
APPLICATION D'UNE MÉTHODE SINACIDE DE DOSAGE DE LA MATIÈRE GRASSE DU LAIT AUX PRODUITS LAITIERS (1)
}

\author{
par \\ J. C. GODFRAIN et D. TRACATELIS
}

Nous avons exposé précédemment comment, en appliquant les principes de SCHAIN, il était possible de doser les matières grasses du lait en utilisant le matériel Gerber. Partant des bons résultats obtenus, nous avons essayé d'étendre la méthode aux produits laitiers (beurre et fromages) ; elle nous a également donné satisfaction et les chiffres expérimentaux sont absolument identiques à ceux donnés par les autres techniques.

Les réactifs sont les mêmes que ceux utilisés antérieurement, aussi ne les décrirons-nous pas à nouveau.

\section{a) Dosage des matières grasses du beurre}

L'appareillage retenu est constitué par le butyromètre spécial Gerber à deux ouvertures, dont le bouchon de l'une d'elles est muni d'un godet qui sert au pesage de la matière à analyser.

La technique est la suivante :

On pèse très exactement 5 grammes de beurre dans le godet fixé au gros bouchon. Celui-ci est mis en place à l'extrémité ouverte correspondante du butyromètre. Par l'autre extrémité, on introduit $8 \mathrm{~cm}^{3}$ du mélange coloré des détergents et de l'eau distillée en quantité suffisante, soit environ, $11 \mathrm{~cm}^{3}$.

Le butyromètre est placé au bain-marie à $85^{\circ}$, l'extrémité supérieure restant toujours débouchée, pendant cinq minutes.

On bouche le butyromètre et on agite fortement pour effectuer un mélange complet des produits contenus.

Le butyromètre est placé, gros bouchon en bas, à nouveau au bain-marie à $85^{\circ}$ pendant cinq à dix minutes, après avoir enlevé le petit bouchon.

On additionne alors d'une certaine quantité d'eau distillée suffisante pour amener la colonne de matière grasse à un niveau convenable, puis, après avoir ajusté le petit bouchon, on centrifuge durant cinq minutes à 1.500 tours/minute.

Après centrifugation, le butyromètre est introduit, toujours dans la même position, au bain-marie réglé à $65-70^{\circ}$, pendant trois minutes. La couche graisseuse apparaît colorée en rose groseille nettement tranchée sur le reste du liquide et la lecture se réalise

(1) Revue de Médecine vétérinaire, 1956, 107, 248. 
d'une façon parfaite, suivant la technique habituelle du procédé Gerber.

Observation. - L'attention doit être portée essentiellement sur l'exactitude de la pesée des 5 grammes de beurre (une erreur de 0 gr. 10 se traduit par un résultat erroné de l'ordre de $1 \mathrm{gr} .6$ ), ainsi que sur la nécessité de faire la lecture sur un butyromètre chauffé ainsi qu'indiqué ci-dessus.

\section{b) Dosage des matières grasses des fromages}

L'appareillage retenu est du même type que dans le dosage précédent, mais la graduation est adaptée.

2 gr. 50 de fromage sont pesés exactement, puis coupés en petits morceaux et placés dans la nacelle fixée au gros bouchon. Celui-ci est introduit dans l'ouverture correspondante du butyromètre. Par l'autre ouverture, on admet $8 \mathrm{~cm}^{3} \mathrm{du}$ mélange coloré ainsi que de l'eau en quantité suffisante.

Le butyromètre, bouché à son extrémité, est agité énergiquement, puis placé au bain-marie réglé à $75-80^{\circ}$, le gros bouchon disposé en bas et le petit bouchon retiré.

Après dix minutes de séjour, le butyromètre est retiré, bouché, agité longuement et replacé au bain-marie pour cinq minutes. Après avoir débouché, on ajoute de l'eau goutte à goutte pour emplir suffisamment.

Le butyromètre, refermé, est centrifugé pendant einq minutes à 1.500 tours/minute.

Le butyromètre est placé, gros bout en bas, au bain-marie à 75-80 pendant trois minutes, puis on procède à la lecture.

Observation. "- Nous renouvelons l'attention à porter sur la pesée et sur la lecture à chaud et ajoutons la nécessité d'une forte agitation.

Les résultats obtenus dans les deux types de dosage ont été en concordance parfaite avec ceux obtenus par d'autres techniques.

\section{BIBLIOGRAPHIE}

[1] J. C. Godfrain. Une méthode sinacide de dosage de la matière grasse du lait utilisant le matériel Gerber. Revue Méd. Vet., 1955, 106, 370 (in Le Lait, 1956, 36, 492).

[2] D. Tracatelis. Application d'une méthode sinacide de dosage de matières grasses à certains produits laitiers. Thèse doct. vét., Toulouse, 1955 .

[3] BAKalor. Dairy Science Abstracts, 1955, 17, n ${ }^{08} 11$ et 12 (article paru après notre première publication et la soutenance de la thèse ci-dessus) avec bibliographie comportant 150 références sur les méthodes rapides de détermination de la teneur en matière grasse du lait. 